\title{
Abstract: Digitale Pathologie für mobile Endgeräte
}

\author{
Hannah Büchner ${ }^{1}$, Ingmar Gergel ${ }^{2}$ \\ ${ }^{1}$ Hochschule Mannheim, mbits imaging $\mathrm{GmbH}$ \\ ${ }^{2}$ Deutsches Krebsforschungszentrum, mbits imaging $\mathrm{GmbH}$ \\ hannahbuechner@mbits.info
}

Digitalisierung spielt im heutigen Zeitalter, besonders in der Medizin, eine entscheidende Rolle. Neben der Radiologie, welche bereits digitalisiert ist, gibt es auch in anderen Fachdisziplinen eine Umstellung bisheriger Workflows. So hat sich auch in den letzten Jahren die digitale Pathologie weiterentwickelt. Anstatt mit einem konventionellen Mikroskop die Glasobjektträger zu betrachten, werden diese zunehmend digitalisiert. Für die Anwendung in der klinischen Routine gibt es aktuell einige Herausforderungen. Einerseits existiert noch kein Standard für ein einheitliches Bildformat, vergleichbar zu DICOM in der Radiologie. Andererseits werden die virtuellen Schnitte durch die verschiedenen Auflösungsstufen sehr groß (bis zu 30 GB), weshalb für die Übertragung ein stabiles Breitbandnetz notwendig ist. Des Weiteren sind derzeit für den Bereitschaftsdienst in der Pathologie keine performanten, mobilen Lösungen für den ortsunabhängigen Zugriff verfügbar.

Im Rahmen einer Bachelorarbeit wurde ein Prototyp entwickelt, welcher ermöglicht, dass verschiedene Dateiformate (.svs, .svslide, .scn, .vms, .vmu, .ndpi, .mrxs, .bif, .tif, .tiff) verarbeitet und standortunabhängig sowohl inner- als auch außerhalb der Arbeitszeiten zur Verfügung stellt. Dieser Prototyp wurde in die Software mRay (CE-zertifiziertes Medizinprodukt IIb) integriert. Um die meisten Bildformate zu unterstützen, wird die OpenSlide-Bibliothek (OpenSource) verwendet. Die eingelesenen Bildinformationen werden in eine eigene Pyramiden-Datenstruktur, bestehend aus mehreren in Bildkacheln eingeteilte Aufösungsebenen, überführt. Um die Speicherplatz-Problematik zu adressieren, werden die Bildkacheln mit verschiedenen Heuristiken (Luminanz, Biomarker) bewertet, sodass relevante Bildareale vorab geladen werden. Dank der Bildkachelung ist es außerdem möglich sehr große Bilder schrittweise zu übertragen und am mobilen Endgerät zu visualisieren.

Vorläufige Ergebnisse zeigen, dass durch die entwickelten Heuristiken und das Vorabladen der volle Funktionsumfang auch mit einer schlechten und keiner Netzwerkverbindung (offline) gewährleistet ist.

Ziel der Arbeit ist es, die digitale Pathologie nicht nur im Bereich von Konsultationen (Schnellschnitt) und Konferenzen, sondern auch in der Lehre durch den mobilen Zugriff zu unterstützen. Es gilt weiterhin zu evaluieren, inwiefern die interdisziplinäre Zusammenarbeit bei Konferenzen durch die Visualisierung mehrerer Bildtypen (MRT, CT, H\&E-gefärbte Schnitte) zu einer Verbesserung in der Patientenbehandlung insgesamt führen kann. 